\title{
Negatively curved bundles in the Igusa stable range
}

\author{
Mauricio Bustamante $\quad$ Francis Thomas Farrell Yi Jiang
}

\begin{abstract}
We use classical results in smoothing theory to extract information about the rational homotopy groups of the space of Riemannian metrics without conjugate points on a high dimensional manifold with hyperbolic fundamental group. As a consequence, we show that spaces of negatively curved Riemannian metrics have in general nontrivial rational homotopy groups. We also show that smooth $M$-bundles over spheres equipped with fiberwise negatively curved metrics represent elements of finite order in the homotopy groups $\pi_{i} B \operatorname{Diff}(M)$ of the classifying space for smooth $M$-bundles, provided $i \ll \operatorname{dim} M$.
\end{abstract}

\section{Introduction}

Let $M$ be a closed smooth manifold. A negatively curved bundle with fiber $M$ is a smooth $M$-bundle $E \rightarrow B$ whose fibers are endowed with continuously varying Riemannian metrics of everywhere negative sectional curvature. This notion has been established in FO10b], where a theory for negatively curved fiber bundles is developed, in the sense that there is a space $\mathcal{T}^{<0}(M)$ with the property that equivalence classes of fiber-homotopically trivial negatively curved bundles over a paracompact space $B$ are in bijective correspondence with homotopy classes of maps $B \rightarrow \mathcal{T}^{<0}(M)$. Here two negatively curved $M$-bundles $E_{1} \rightarrow B$ and $E_{2} \rightarrow$ $B$ are equivalent if there exists a negatively curved $M$-bundle $\mathcal{E}$ over $B \times[0,1]$ such that $\mathcal{E}$ restricted to $B \times\{i\}$ is fiberwise isometric to $E_{i}, i=1,2$ (see [FO10b, p.1399]). From a purely topological point of view, fiber homotopically trivial smooth bundles over $B$ with fiber a negatively curved manifold $M$ are classified, up to bundle equivalence, by homotopy classes of maps $B \rightarrow B \operatorname{Diff}_{0}(M)$, where $\operatorname{Diff}_{0}(M)$ is the group of all diffeomorphisms of $M$ which are homotopic to the identity on $M$. Thus there is a natural "forgetful" map

$$
\mathfrak{F}: \mathcal{T}^{<0}(M) \rightarrow B \operatorname{Diff}_{0}(M) .
$$

One then wonders how much these two bundle theories differ, and this is the theme of this paper. This question has been addressed already by Farrell and Ontaneda in [FO09, FO10a, FO10b]. A remarkable observation is that the homotopy fiber of the forgetful map $\mathfrak{F}$ can be identified with the space $\operatorname{MET}^{<0}(M)$ of all negatively curved metrics on $M$, so that we have a homotopy fibration

$$
\operatorname{MET}^{<0}(M) \rightarrow \mathcal{T}^{<0}(M) \stackrel{\mathfrak{F}}{\rightarrow} B \operatorname{Diff}_{0}(M) .
$$

Farrell and Ontaneda FO10a, have shown that the space of negatively curved metrics on $M$ is highly non-connected if the dimension of $M$ is sufficiently large, thus the two bundle theories are fundamentally different. This difference was captured in their main theorem by elements of finite order in the homotopy groups of $\mathrm{MET}^{<0}(M)$. Nevertheless, one could still hope that the two theories were "rationally equivalent" (this kind of phenomenon occurs for example in the theory of stable vector bundles and stable topological $\mathbb{R}^{n}$-bundles. Indeed, $B \mathrm{O}$ and $B$ Top are only rationally equivalent). Our main result establishes that these two bundle theories are inequivalent even if one decides to neglect torsion, at least in a range of dimensions called the 
Igusa stable range. This is done by showing that the rational homotopy groups of $\operatorname{MET}^{<0}(M)$ are in general nontrivial.

Let us now assume that $M$ is a closed manifold that supports a Riemannian metric $g$ without conjugate points, that is for any geodesic $\gamma$ of $M$ no two points are conjugate along $\gamma$ (e.g. nonpositively curved metrics, or metrics with geodesic flow of Anosov type). In particular $M$

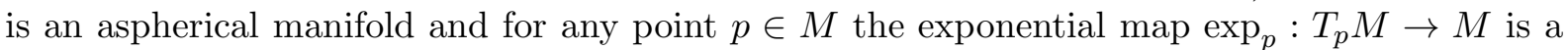
(universal) covering map. Let $\operatorname{MET}^{\mathcal{N C}}(M)$ denote the space of all such Riemannian metrics on $M$. Then given any self-diffeomorphism $f: M \rightarrow M$ of $M$, we define the push-forward metric $g^{\prime}$ on $M$ to be the unique Riemannian metric such that $f:(M, g) \rightarrow\left(M, g^{\prime}\right)$ is an isometry. We denote the push-forward metric by $f_{*} g$. This gives rise to an orbit map $\Phi^{g}: \operatorname{Diff}_{0}(M) \rightarrow$ $\operatorname{MET}^{\mathcal{N C}}(M)$, defined by $\Phi^{g}(f)=f_{*} g$, and the corresponding map in homotopy groups

$$
\Phi_{*}^{g}: \pi_{i} \operatorname{Diff}_{0}(M) \rightarrow \pi_{i} \operatorname{MET}^{\mathcal{N C}}(M) .
$$

Theorem 1. Let $(M, g)$ be a closed Riemannian $n$-manifold without conjugate points. Assume that the fundamental group of $M$ is hyperbolic. Then for all $1<i<\min \left\{\frac{n-10}{2}, \frac{n-8}{3}\right\}$ the map

$$
\Phi_{*}^{g}: \pi_{i}\left(\operatorname{Diff}_{0}(M), i d\right) \otimes \mathbb{Q} \rightarrow \pi_{i}\left(\operatorname{MET}^{\mathcal{N C}}(M), g\right) \otimes \mathbb{Q}
$$

is injective.

For the definition of a hyperbolic group we refer the reader to [BH99] or [Gro87].

Note that Theorem 1 does give elements of infinite order in $\pi_{i} \operatorname{MET}^{\mathcal{N C}}(M)$ in view of the following result of Farrell and Hsiang. First recall that a closed manifold $M$ satisfies the strong Borel conjecture if for all $k \geq 0$, every self-homotopy equivalence of pairs $\left(M \times D^{k}, M \times S^{k-1}\right) \rightarrow$ $\left(M \times D^{k}, M \times S^{k-1}\right)$ which is a homeomorphism when restricted to the boundary $M \times S^{k-1}$ is homotopic (relative to the boundary) to a homeomorphism.

Theorem (Farrell-Hsiang [FH78). Let $M$ be a closed aspherical smooth $n$-manifold that satisfies both the strong Borel conjecture and Conjecture 2 in [FH78, p.326]. Then if $0<i<$ $\min \left\{\frac{n-7}{2}, \frac{n-4}{3}\right\} 1$,

$$
\pi_{i} \operatorname{Diff}_{0}(M) \otimes \mathbb{Q}= \begin{cases}\bigoplus_{j=1}^{\infty} H_{(i+1)-4 j}(M, \mathbb{Q}) & \text { if } n \text { is odd } \\ 0 & \text { if } n \text { is even }\end{cases}
$$

Some important classes of manifolds relevant to the subject of this paper which satisfy both the strong Borel conjecture and Conjecture 2 in [FH78, p.326] are: closed negatively curved manifolds [FJ89] or more generally closed nonpositively curved manifolds [FJ93 and closed aspherical manifolds with hyperbolic fundamental group [BLR08, BL12. For all those, the calculation (2) holds.

In the case of a negatively curved Riemannian manifold $(M, g)$ the orbit map $\Phi^{g}$ factors through the inclusion

$$
\operatorname{MET}^{<0}(M) \hookrightarrow \operatorname{MET}^{\mathcal{N C}}(M),
$$

and since the fundamental group of $M$ is hyperbolic, we have:

\footnotetext{
${ }^{1}$ By the time Farrell and Hsiang obtained this result the stability range was roughly $i<n / 6$. Later Igusa improved this range to the one stated here. See Igu88 and Igu02, p.252].
} 
Corollary 1.1. Let $(M, g)$ be a closed negatively curved Riemannian $n$-manifold. Then for all $1<i<\min \left\{\frac{n-10}{2}, \frac{n-8}{3}\right\}$ the map

$$
\Phi_{*}^{g}: \pi_{i}\left(\operatorname{Diff}_{0}(M), i d\right) \otimes \mathbb{Q} \rightarrow \pi_{i}\left(\operatorname{MET}^{<0}(M), g\right) \otimes \mathbb{Q}
$$

is injective.

Remark 1. Similar corollaries can be obtained in the same way for various spaces of Riemannian metrics. Some other examples are: the space $\operatorname{MET}^{\leq 0}(M)$ of nonpositively curved Riemannian metrics on a closed negatively curved manifold $M$ (considered already in [FO15]), and the space $\operatorname{MET}^{\mathcal{A}}(M)$ of Riemannian metrics with geodesic flow of Anosov type (see [Kli74] for the nonconjugate points condition and Rug03, Rug94 for hyperbolicity of the fundamental group).

Corollary 1.1 together with the long exact sequence in homotopy groups for the fibration (11) imply that, in the Igusa stable range, every negatively curved bundle over an $(i+1)$-sphere has finite order (viewed as an element in $\pi_{i+1} B \operatorname{Diff}_{0}(M)$ ). More precisely:

Corollary 1.2. Let $(M, g)$ be a closed negatively curved Riemannian $n$-manifold. Then for all $2<i<\min \left\{\frac{n-10}{2}, \frac{n-8}{3}\right\}$ there is a short exact sequence

$$
0 \rightarrow \pi_{i} \operatorname{Diff}_{0}(M) \otimes \mathbb{Q} \rightarrow \pi_{i} \operatorname{MET}^{<0}(M) \otimes \mathbb{Q} \rightarrow \pi_{i} \mathcal{T}^{<0}(M) \otimes \mathbb{Q} \rightarrow 0 .
$$

In particular, the forgetful map $\mathfrak{F} \otimes i d_{\mathbb{Q}}: \pi_{i+1} \mathcal{T}^{<0}(M) \otimes \mathbb{Q} \rightarrow \pi_{i+1} B \operatorname{Diff}_{0}(M) \otimes \mathbb{Q}$ is trivial for all $1<i<\min \left\{\frac{n-10}{2}, \frac{n-8}{3}\right\}$.

Instead of looking at smooth negatively curved Riemannian metrics, one could consider the weaker notion of a CAT(-1) metric on the closed smooth manifold $M$, that is metrics on $M$ with the property that every geodesic triangle is "thinner" than the corresponding comparison triangle in the hyperbolic plane (for a precise definition see [BH99]). Let $\mathrm{CAT}^{-1}(M)$ be the space of all such metrics on $M$ with the $C^{0}$-topology. There is a continuous map $\beta$ : $\mathrm{MET}^{<-1}(M) \rightarrow$ $\mathrm{CAT}^{-1}(M)$ given by sending each negatively curved metric $M$ to the induced distance function on $M$. Here $\mathrm{MET}^{<-1}(M)$ is the space of all metrics on $M$ with curvature less than -1 .

Corollary 1.3. There exist an $i>0$, a closed negatively curved manifold $M$ and a non-trivial smooth $M$-bundle over the $(i+1)$-sphere $S^{i+1}$, which admits a continuously varying family of CAT $(-1)$ metrics on the fibers and it is not a negatively curved bundle.

Proof. Take $\left(M^{n}, g\right)$ to be an odd-dimensional closed negatively curved manifold such that $H_{(i+1)-4 j}(M, \mathbb{Q}) \neq 0$ for some $1<i<\min \left\{\frac{n-10}{2}, \frac{n-8}{3}\right\}$ and some $j \geq 1$ (e.g. for $i=3$ and $j=1$, any connected 19-dimensional negatively curved manifold satisfies this condition). By (2), there is a map $f: S^{i} \rightarrow \operatorname{Diff}_{0}(M)$ which represents a non-trivial class $[f]$ in $\pi_{i} \operatorname{Diff}_{0}(M) \otimes \mathbb{Q}$, but maps to zero in $\pi_{i} \operatorname{Top}_{0}(M)$. This is possible since $\pi_{i} \operatorname{Top}_{0}(M)$ is a torsion abelian group when $1<i<\min \left\{\frac{n-7}{2}, \frac{n-4}{3}\right\}$ by [FJ93] (see also [Far02]). Now perform the the clutching construction, that is glue two copies of $D^{i+1} \times M$ with their boundaries identified by $(z, y) \mapsto(z, f(z)(y))$, for $(z, y) \in \partial D^{i+1} \times M$. This gives rise to non-trivial smooth $M$-bundle over the $(i+1)$-sphere. This bundle is not a negatively curved bundle. Indeed, if it were, then $[f]$ would be in the image of the forget map $\mathfrak{F} \otimes i d_{\mathbb{Q}}: \pi_{i+1} \mathcal{T}^{<0}(M) \otimes \mathbb{Q} \rightarrow \pi_{i+1} B \operatorname{Diff}_{0}(M) \otimes \mathbb{Q}$. But this is not possible by Corollary 1.2. To show that the bundle admits a continuously varying family of $\mathrm{CAT}(-1)$ metrics, it suffices to show that the map $S^{i} \rightarrow \mathrm{CAT}^{-1}(M)$ given by $z \mapsto \beta\left(f(z)_{*} g\right)$ extends over the $(i+1)$-disc. To see this, note that $\operatorname{Top}_{0}(M)$ acts continuously on $\operatorname{CAT}^{-1}(M)$ by 
pushing-forward the distance function induced by $g$. One can form the following commutative diagram

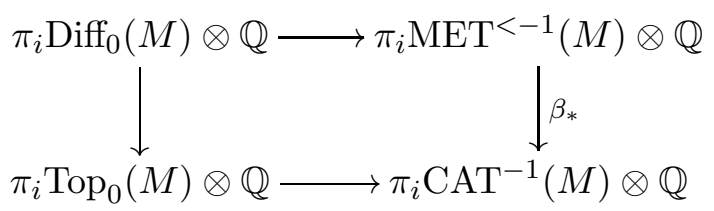

As noted above, the lower left group vanishes for $1<i<\min \left\{\frac{n-7}{2}, \frac{n-4}{3}\right\}$. This completes the proof of the corollary.

\section{Comments on other related work}

1. As mentioned before Farrell and Ontaneda [FO10a] obtain torsion elements in the homotopy groups of the space of negatively curved Riemannian metrics. Their method would never give infinite order elements because it depends strongly on the existence of nontrivial elements in the homotopy groups of the space of stable topological pseudoisotopies of the circle. But in Igusa's stable range, those are torsion groups Wal78.

2. Farrell and Ontaneda [FO09] find non-trivial elements of finite order in $\pi_{i} \mathcal{T}^{<0}(M)$, when $i \ll \operatorname{dim} M$, and $M$ is a real hyperbolic manifold. But again their method fails to give information about infinite order elements in these homotopy groups. The reason here is that their construction relies on finding nonzero elements in $\pi_{i} \operatorname{Diff}\left(D^{n}, \partial\right)$ which have preimages under the Gromoll map $\pi_{i+1} \operatorname{Diff}\left(D^{n-1}, \partial\right) \rightarrow \pi_{i} \operatorname{Diff}\left(D^{n}, \partial\right)$, where $\operatorname{Diff}\left(D^{n}, \partial\right)$ is the group of diffeomorphisms of a closed disc $D^{n}$ which are the identity on the boundary. But in the Igusa stability range, this map is rationally trivial [FH78]. Nonetheless elements of infinite order in $\pi_{i} \mathcal{T}^{<0}(M)$ can be obtained if we look outside Igusa's stable range. We elaborate on this in [BFJ18].

Our main tool to prove Theorem 1 is Morlet's comparison theorem, which we recall in Section 2. In Section 3 we reduce Theorem 1 to two lemmas: one of pure topological nature (proved in Section 4), and one that incorporates the geometry of $(M, g)$ (proved in the last section).

\section{Acknowledgements}

M. Bustamante is supported by the DFG Priority Programme Geometry at Infinity (SPP 2026): Spaces and moduli spaces of Riemannian metrics with curvature bounds on compact and noncompact manifolds. He acknowledges the hospitality of the Yau Mathematical Sciences Center at Tsinghua University in Beijing, where he was a postdoc when most of the results of this paper were obtained.

\section{Background and notation}

Throughout this paper, $\operatorname{Top}(X)$ denotes the group of self-homeomorphisms of a topological space $X$, endowed with the compact open topology. If $X$ is a closed smooth manifold then $\operatorname{Diff}(X)$ denotes the group of smooth self-diffeomorphisms of $X$ with the smooth topology. We denote by $\operatorname{Top}_{0}(X)$ (resp. $\left.\operatorname{Diff}_{0}(X)\right)$ the subgroup of $\operatorname{Top}(X)$ (resp. Diff $(X)$ ) consisting of all those self-homeomorphisms (resp. self-diffeomorphisms) of $X$ which are homotopic to the identity map. Also, $\operatorname{Top}(n)$ will denote the group of homeomorphisms of $\mathbb{R}^{n}$ with the compact open topology. We keep the customary notation $\mathrm{O}(n)$ for the group of orthogonal (w.r.t. Euclidean metric) transformations of $\mathbb{R}^{n}$ with its usual topology. 


\subsection{Morlet's comparison theorem}

Let $M$ be a closed smooth $n$-dimensional manifold. Let $\operatorname{TOP}(M)$ and $\operatorname{DIFF}(M)$ denote the singular and smooth singular complex of $\operatorname{Top}(M)$ and $\operatorname{Diff}(M)$ respectively. These are simplicial sets whose geometric realizations are weakly homotopy equivalent to $\operatorname{Top}(M)$ and $\operatorname{Diff}(M)$ respectively. A $k$-simplex of $\mathbf{T O P}(M)$ (resp. $\mathbf{D I F F}(M))$ is a homeomorphism (resp. diffeomorphism) $\Delta^{k} \times M \rightarrow \Delta^{k} \times M$ which commutes with the projection to the standard $k$ simplex $\Delta^{k}$. The corresponding singular and smooth singular complexes of $\operatorname{Top}_{0}(M)$ and $\operatorname{Diff}_{0}(M)$ are denoted by $\mathbf{T O P}_{0}(M)$ and $\mathbf{D} \mathbf{I F F}_{0}(M)$ respectively.

One advantage of working in this setting is that the simplicial group $\mathbf{D I F F}_{0}(M)$ acts freely on $\mathbf{T} \mathbf{O P}_{0}(M)$ and so the quotient $\frac{\mathbf{T O P}_{0}(M)}{\mathbf{D I F F}_{0}(M)}$ is naturally a simplicial set, in fact a Kan complex. Furthermore there is a Kan fibration

$$
\operatorname{DIFF}_{0}(M) \rightarrow \operatorname{TOP}_{0}(M) \rightarrow \frac{\operatorname{TOP}_{0}(M)}{\operatorname{DIFF}_{0}(M)}
$$

which gives rise to the following long exact sequence of homotopy groups

$$
\cdots \rightarrow \pi_{i+1} \operatorname{Top}_{0}(M) \rightarrow \pi_{i+1} \frac{\operatorname{Top}_{0}(M)}{\operatorname{Diff}_{0}(M)} \stackrel{d_{*}}{\rightarrow} \pi_{i} \operatorname{Diff}_{0}(M) \rightarrow \pi_{i} \operatorname{Top}_{0}(M) \rightarrow \cdots
$$

where we denote by $\frac{\operatorname{Top}_{0}(M)}{\operatorname{Diff}_{0}(M)}$ the geometric realization of $\frac{\mathbf{T O P}_{0}(M)}{\operatorname{DIFF}_{0}(M)}$, and the homotopy groups of $\left|\mathbf{T O P}_{0}\right|$ (resp. $\left|\mathbf{D I F F}_{0}\right|$ ) are identified with those of $\operatorname{Top}_{0}(M)$ (resp. Diff $0(M)$ ).

Assume now that $M$ comes equipped with a Riemannian metric, and regard its tangent bundle $T M$ as a Euclidean vector bundle. There is an associated (right) principal $\mathrm{O}(n)$-bundle $P M \rightarrow M$.

Note that $\mathrm{O}(n)$ acts on the left on the coset space $\frac{\operatorname{Top}(n)}{\mathrm{O}(n)}$. Thus we can form the balanced product

$$
\mathcal{B}_{n}(M):=P M \times \mathrm{O}(n) \frac{\mathrm{Top}(n)}{\mathrm{O}(n)},
$$

which is a fiber bundle over $M$ with fiber $\frac{\operatorname{Top}(n)}{\mathrm{O}(n)}$.

The space (with the compact-open topology) of sections of $\mathcal{B}_{n}(M)$ is denoted by $\Gamma\left(\mathcal{B}_{n}(M)\right)$. Note that $\Gamma\left(\mathcal{B}_{n}(M)\right)$ has a preferred element $s_{0}: M \rightarrow \mathcal{B}_{n}(M)$ given by the $\mathrm{O}(n)$-invariant point id $\mathrm{O}(n) \in \operatorname{Top}(n) / \mathrm{O}(n)$.

The space $\frac{\operatorname{Top}_{0}(M)}{\operatorname{Diff}_{0}(M)}$ can be related to the space of sections $\Gamma\left(\mathcal{B}_{n}(M)\right)$. We briefly recall how this is done, following the ideas of [BL74]. Consider simplicial sets $\overline{\mathbf{R}}^{T}(M) \supset \overline{\mathbf{R}}^{d}(M) \supset \overline{\mathbf{R}}^{o}(M)$ of topological, linear and orthogonal representations of the tangent bundle of $M$ respectively. That is, a $k$-simplex of $\overline{\mathbf{R}}^{T}(M)$ is a topological $\mathbb{R}^{n}$-bundle isomorphism $\Delta^{k} \times T M \rightarrow \Delta^{k} \times T M$ which leaves invariant the image of the zero section, and which commutes with the projection to $\Delta^{k}$ and covers some homeomorphism $\Delta^{k} \times M \rightarrow \Delta^{k} \times M$. The $k$-simplices of $\overline{\mathbf{R}}^{d}(M)$ and $\overline{\mathbf{R}}^{o}(M)$ are defined similarly but the maps $\Delta^{k} \times T M \rightarrow \Delta^{k} \times T M$ are now vector bundle isomorphisms and vector bundle isomorphisms which are fiberwise isometries, respectively. We also consider a simplicial set $\overline{\mathbf{R}}^{t}(M)$ whose $k$-simplices are germs of topological microbundle isomorphisms of $\Delta^{k} \times T M$ which commute with the projection onto $\Delta^{k}$, and cover some homeomorphism $\Delta^{k} \times M \rightarrow \Delta^{k} \times M$.

Simplicial sets $\underline{\mathbf{R}}^{t}(M), \underline{\mathbf{R}}^{T}(M), \underline{\mathbf{R}}^{d}(M)$ and $\underline{\mathbf{R}}^{o}(M)$ are defined analogously, the only difference is that the isomorphisms $\Delta^{k} \times T M \rightarrow \Delta^{k} \times T M$ must cover the identity map on $\Delta^{k} \times M$.

Now recall that the tangent microbundle of $M$ is the diagram $M \stackrel{\Delta}{\rightarrow} M \times M \stackrel{p r_{1}}{\longrightarrow} M$, where $\Delta$ is the diagonal map and $p r_{1}$ is the projection onto the first factor. Since we have fixed a 
Riemannian metric on $M$, the exponential map gives an isomorphism between $T M$ and the tangent microbundle of $M$ (see e.g. [Mil64, p.56-58]). Thus, via this isomorphism, any selfhomeomorphism $f: M \rightarrow M$ induces, by taking $f \times f$, a microbundle automorphism of $T M$ which is called the topological derivative of $f$. This gives rise to a simplicial map (c.f. [BL77, p.453])

$$
\delta: \frac{\mathbf{T O P}_{0}(M)}{\mathbf{D I F F}_{0}(M)} \rightarrow \overline{\mathbf{R}}^{t}(M) / \overline{\mathbf{R}}^{d}(M)
$$

Morlet's comparison theorem is the statement that the topological derivative $\delta$ induces an injective map on connected components and an isomorphism on higher homotopy groups, provided $M$ is a closed manifold of dimension $\neq 4$ [BL74, Proposition 4.3].

To complete the picture, we relate $\overline{\mathbf{R}}^{t}(M) / \overline{\mathbf{R}}^{d}(M)$ to the space of sections $\Gamma\left(\mathcal{B}_{n}(M)\right.$ ) (or rather its singular simplicial complex $\mathbf{S} \Gamma\left(\mathcal{B}_{n}(M)\right)$ ) via the following two lemmas, whose proofs are an elaboration of the argument given by Burghelea and Lashof in [BL74, Theorem $\left.4.2\left(1^{t}\right)\right]$ :

Lemma 2. The simplicial sets $\overline{\mathbf{R}}^{t}(M) / \overline{\mathbf{R}}^{d}(M)$ and $\underline{\mathbf{R}}^{T}(M) / \underline{\mathbf{R}}^{o}(M)$ are weakly homotopy equivalent.

Lemma 3. There exits a map $S: \underline{\mathbf{R}}^{T}(M) / \underline{\mathbf{R}}^{o}(M) \rightarrow \mathbf{S} \Gamma\left(\mathcal{B}_{n}(M)\right)$ which induces an injective map on connected components and a weak homotopy equivalence on any connected component.

The proofs of the lemmas are deferred to the Appendix below.

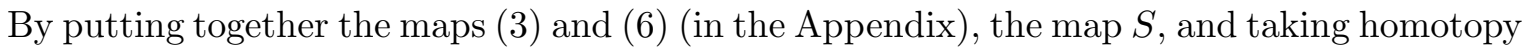
groups we obtain a map

$$
\mu_{*}: \pi_{i} \frac{\operatorname{Top}_{0}(M)}{\operatorname{Diff}_{0}(M)} \rightarrow \pi_{i} \Gamma\left(\mathcal{B}_{n}(M)\right),
$$

which is injective for $i=0$ and an isomorphism for all $i>0$ for every component that is hit. This is called Morlet's isomorphism.

Remark 2. There is a version of Morlet's theorem for smooth compact manifolds with boundary (see BL74). In particular it follows from this and the Alexander trick, that there is a weak homotopy equivalence $\operatorname{Diff}\left(D^{n}, \partial\right) \rightarrow \Omega^{n+1} \frac{\operatorname{Top}(n)}{\mathrm{O}(n)}$, when $n \neq 4$.

\section{Outline of the proof of Theorem 1}

Let $D^{n}$ denote a closed unit disc in $\mathbb{R}^{n}$ centered at the the origin and let $\operatorname{int} D^{n}$ be its interior. Observe that the balanced product

$$
\mathcal{B}_{n}^{D}(M)=P M \times{ }_{\mathrm{O}(n)} \frac{\operatorname{Top}\left(D^{n}\right)}{\mathrm{O}(n)}
$$

is a fiber bundle over $M$ with fiber $\frac{\operatorname{Top}\left(D^{n}\right)}{\mathrm{O}(n)}$ which has a preferred section, namely the obvious map $s_{1}: M=P M \times{ }_{\mathrm{O}(n)} \frac{\mathrm{O}(n)}{\mathrm{O}(n)} \rightarrow P M \times \mathrm{O}_{(n)} \frac{\mathrm{Top}\left(D^{n}\right)}{\mathrm{O}(n)}$.

Lemma 4. Let $M$ be a closed smooth $n$-dimensional manifold with $n$ odd. Then for all $1<$ $i<\min \left\{\frac{n-8}{2}, \frac{n-5}{3}\right\}$

$$
\pi_{i}\left(\Gamma\left(\mathcal{B}_{n}^{D}(M)\right), s_{1}\right) \otimes \mathbb{Q}=0
$$


Throughout we fix a homeomorphism $\varphi: \operatorname{int} D^{n} \rightarrow \mathbb{R}^{n}$ defined by

$$
\varphi(v)=\frac{v}{1-\|v\|} .
$$

Conjugation by $\varphi$ defines a continuous injective $\operatorname{map} \operatorname{int}_{\varphi}: \operatorname{Top}\left(D^{n}\right) \rightarrow \operatorname{Top}(n)$, that is

$$
\operatorname{int}_{\varphi}(f)=\left.\varphi \circ f\right|_{\operatorname{int}\left(D^{n}\right)} \circ \varphi^{-1}
$$

Clearly this map is equivariant with respect to both the right and the left action of $\mathrm{O}(n)$, and hence it induces a left equivariant map between orbit spaces that we keep denoting by $\operatorname{int}_{\varphi}: \frac{\operatorname{Top}\left(D^{n}\right)}{\mathrm{O}(n)} \rightarrow \frac{\operatorname{Top}(n)}{\mathrm{O}(n)}$. Furthermore $\operatorname{int}_{\varphi}$ gives rise to a map between spaces of sections

$$
\operatorname{int}_{\varphi}:\left(\Gamma\left(\mathcal{B}_{n}^{D}(M)\right), s_{1}\right) \rightarrow\left(\Gamma\left(\mathcal{B}_{n}(M)\right), s_{0}\right) .
$$

Lemma 5. Let $g \in \operatorname{MET}^{\mathcal{N C}}(M)$. Assume that $\pi_{1} M$ is a hyperbolic group. Then for all $i \geq 0$ and $y \in \operatorname{ker} \Phi_{*}^{g} \circ d_{*} \subset \pi_{i+1} \frac{\operatorname{Top}_{0}(M)}{\operatorname{Diff}_{0}(M)}$, there exists $\bar{y} \in \pi_{i+1} \Gamma\left(\mathcal{B}_{n}^{D}(M)\right)$ such that

$$
\operatorname{int}_{\varphi *}(\bar{y})=\mu_{*}(y) \in \pi_{i+1} \Gamma\left(\mathcal{B}_{n}(M)\right)
$$

Proof of Theorem 1] assuming Lemma 4 and Lemma 5. If $n$ is even the orbit map is obviously rationally injective by (21). If $n$ is odd then Lemma 4 and Lemma 5 imply that $\operatorname{ker}\left(\Phi_{*}^{g} \circ d_{*}\right) \otimes i d_{\mathbb{Q}}=0$ for $1<i<\min \left\{\frac{n-10}{2}, \frac{n-8}{3}\right\}$. But it is known (see e.g. [Far02], [FJ93]) that $\pi_{i} \operatorname{Top}_{0}(M) \otimes \mathbb{Q}=0$ provided $M$ is aspherical and satisfies the strong Borel conjecture and $1<$ $i<\min \left\{\frac{n-7}{2}, \frac{n-4}{3}\right\}$. That manifolds with hyperbolic fundamental group satisfy both the strong Borel conjecture and Conjecture 2 in [FH78, p.326], follows from [BLR08] and [BL12. Hence $d_{*} \otimes i d_{\mathbb{Q}}: \pi_{i+1} \frac{\operatorname{Top}_{0}(M)}{\operatorname{Diff}_{0}(M)} \otimes \mathbb{Q} \rightarrow \pi_{i} \operatorname{Diff}_{0}(M) \otimes \mathbb{Q}$ is an isomorphism when $1<i<\min \left\{\frac{n-9}{2}, \frac{n-7}{3}\right\}$. Therefore the orbit map $\Phi_{*}^{g} \otimes i d_{\mathbb{Q}}: \pi_{i} \operatorname{Diff}_{0}(M) \otimes \mathbb{Q} \rightarrow \pi_{i} \operatorname{MET}^{\mathcal{N C}}(M) \otimes \mathbb{Q}$ must be injective for $1<i<\min \left\{\frac{n-10}{2}, \frac{n-8}{3}\right\}$.

The rest of the paper is devoted to the proof of Lemma 4 and Lemma 5 .

\section{Proof of Lemma 4}

The rationalization of a nilpotent space $X$ will be denoted by $X_{(0)}$. A map $f: X \rightarrow Y$ between nilpotent spaces is rationally $k$-connected if the induced map $f_{(0)}: X_{(0)} \rightarrow Y_{(0)}$ between their rationalizations is $k$-connected.

We have the following general fact (compare [BL77, Lemma 3.10]).

Lemma 6. Let $E_{i} \rightarrow B, i=1,2$ be two fibrations over a finite CW-complex $B$ whose fibers $F_{i}$, $i=1,2$, over a point $* \in B$ are simply connected spaces. Let $\beta: E_{1} \rightarrow E_{2}$ be a continuous map over the identity on $B$ such that the restriction $\beta: F_{1} \rightarrow F_{2}$ is rationally $k$-connected. Then the induced map $\Gamma(\beta): \Gamma\left(E_{1}\right) \rightarrow \Gamma\left(E_{2}\right)$ between section spaces is rationally $(k-\operatorname{dim} B)$-connected. In particular, if $\pi_{j} F_{1} \otimes \mathbb{Q}=0$ for $1<j \leq \ell$, then $\pi_{j} \Gamma\left(E_{1}\right) \otimes \mathbb{Q}=0$ for $1<j \leq \ell-\operatorname{dim} B$.

Proof. Assume first that the fibers $F_{i}, i=1,2$ have the homotopy type of a CW-complex. Fiberwise rationalize the two fibrations (see [BK72, p.40] or [Lle85]) to obtain another pair of fibrations $F_{i(0)} \rightarrow \mathcal{E}_{i} \rightarrow B$ over $B$ and a fiber preserving map $\mathcal{E}_{1} \rightarrow \mathcal{E}_{2}$. Since the restriction $\beta_{(0)}: F_{1(0)} \rightarrow F_{2(0)}$ of this map is assumed to be $k$-connected, we have that the induced map $\Gamma\left(\mathcal{E}_{1}\right) \rightarrow \Gamma\left(\mathcal{E}_{2}\right)$ between spaces of sections is $(k-\operatorname{dim} B)$-connected ([BL77, Lemma 3.10]). 
The lemma now follows in this case since $\Gamma\left(\mathcal{E}_{i}\right), i=1,2$ is nilpotent and fiberwise localization commutes with taking spaces of sections, as shown by Møller in [Ml87, Theorem 5.3].

In order to handle the case when the fibers $F_{i}$ may not have the homotopy type of a CWcomplex, let $\mathbf{S}$ be the functor that assigns to a space its singular complex. We let $\mathcal{G}_{i} \rightarrow B$ be the pull-back of the fibration $\left|\mathbf{S} E_{i}\right| \rightarrow|\mathbf{S} B|$ along a fixed homotopy inverse $\varphi$ of the natural map $\psi:|\mathbf{S} B| \rightarrow B$. Then there is a commutative diagram

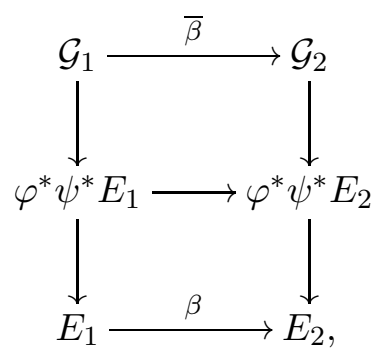

where the vertical maps cover the identity map on $B$ and restrict to weakly homotopy equivalences on the fibers. Since the restriction $\beta: F_{1} \rightarrow F_{2}$ is rationally $k$-connected, so is the restriction of $\bar{\beta}$ to the fiber, and hence the induced map $\Gamma(\bar{\beta}): \Gamma\left(\mathcal{G}_{1}\right) \rightarrow \Gamma\left(\mathcal{G}_{2}\right)$ is rationally $(k-\operatorname{dim} B)$-connected by the previous case. Consequently $\Gamma(\beta)$ is rationally $(k-\operatorname{dim} B)$ connected.

Fix a point $* \in \partial D^{n} \subset D^{n}$. Let $\operatorname{Top}\left(D^{n}, *\right)$ denote the (closed) subgroup of $\operatorname{Top}\left(D^{n}\right)$ consisting of all homeomorphisms $f: D^{n} \rightarrow D^{n}$ such that $f(*)=*$. Note that $\mathrm{O}(n-1)$, identified with the subgroup of $\mathrm{O}(n)$ that fixes $*$, acts naturally (on the right) on $\operatorname{Top}\left(D^{n}, *\right)$

Lemma 7. The orbit spaces $\frac{\operatorname{Top}\left(D^{n}\right)}{\mathrm{O}(n)}$ and $\frac{\operatorname{Top}\left(D^{n}, *\right)}{\mathrm{O}(n-1)}$ are homeomorphic.

Proof. The subgroup inclusion $\operatorname{Top}\left(D^{n}, *\right) \subset \operatorname{Top}\left(D^{n}\right)$ induces a continuous injective map $\frac{\operatorname{Top}\left(D^{n}, *\right)}{\mathrm{O}(n-1)} \stackrel{\iota}{\rightarrow} \frac{\operatorname{Top}\left(D^{n}\right)}{\mathrm{O}(n)}$. Note that for each $f \in \operatorname{Top}\left(D^{n}\right)$, there exists an orthogonal transformation $A \in \mathrm{O}(n)$ such that $A(f(*))=*$. It is easy to see that the assignment $f \mapsto A \circ f$ induces an inverse to $\iota$. Thus $\iota$ is a continuous bijection. But since the orbit map $\operatorname{Top}\left(D^{n}\right) \rightarrow \frac{\operatorname{Top}\left(D^{n}\right)}{\mathrm{O}(n)}$ is a closed map, $\iota$ is a closed map as well. Hence it is a homeomorphism.

Proof of Lemma 4: Since the restriction map $\operatorname{Top}\left(D^{n}, *\right) \rightarrow \operatorname{Top}\left(S^{n-1}, *\right)$ is a homotopy equivalence (coning provides a homotopy inverse, by the Alexander trick), the induced map $\frac{\operatorname{Top}\left(D^{n}, *\right)}{\mathrm{O}(n-1)} \rightarrow \frac{\operatorname{Top}\left(S^{n-1}, *\right)}{\mathrm{O}(n-1)}$ is a weak homotopy equivalence, which can be seen by applying the Five Lemma to long exact sequences in homotopy associated to the fibrations $\operatorname{Top}\left(D^{n}, *\right) \rightarrow \frac{\operatorname{Top}\left(D^{n}, *\right)}{\mathrm{O}(n-1)}$ and $\operatorname{Top}\left(S^{n-1}, *\right) \rightarrow \frac{\operatorname{Top}\left(S^{n-1}, *\right)}{\mathrm{O}(n)}$. Thus, as $\frac{\operatorname{Top}\left(D^{n}\right)}{\mathrm{O}(n)}$ is homeomorphic to $\frac{\operatorname{Top}\left(D^{n}, *\right)}{\mathrm{O}(n-1)}$, it is weakly homotopy equivalent to $\frac{\operatorname{Top}\left(S^{n-1}, *\right)}{\mathrm{O}(n-1)} \simeq \frac{\operatorname{Top}(n-1)}{\mathrm{O}(n-1)}$, where the last identification is done via the stereographic projection. It then follows from [KS77, Essay V $\S 5]$ and [KM63], that $\frac{\operatorname{Top}\left(D^{n}\right)}{\mathrm{O}(n)}$ is simply connected and $\pi_{j} \frac{\operatorname{Top}\left(D^{n}\right)}{\mathrm{O}(n)} \otimes \mathbb{Q}=0$ for all $1 \leq j \leq n$. Now, by Morlet's comparison theorem we have that for all $j \geq n-1$

$$
\pi_{j+1} \frac{\operatorname{Top}(n-1)}{\mathrm{O}(n-1)} \simeq \pi_{j-(n-1)} \operatorname{Diff}\left(D^{n-1}, \partial\right) .
$$


But Farrell and Hsiang [FH78] showed that when $n$ is odd $\pi_{j-(n-1)} \operatorname{Diff}\left(D^{n-1}, \partial\right) \otimes \mathbb{Q}=0$ if $0<$ $j-(n-1)<\min \left\{\frac{n-8}{2}, \frac{n-5}{3}\right\}$. Hence $\pi_{i} \operatorname{Top}\left(D^{n}\right) / \mathrm{O}(n) \otimes \mathbb{Q}=0$ for all $1 \leq i<n+\min \left\{\frac{n-8}{2}, \frac{n-5}{3}\right\}$. The result now follows from Lemma 6 .

\section{Proof of Lemma 5}

Let $(M, g)$ be a closed Riemannian manifold without conjugate points. Its tangent bundle $T M \stackrel{\pi}{\rightarrow} M$ is regarded as a Euclidean vector bundle with respect to the metric $g$ (see [MS74, p.21-22]).

The tangent disc bundle $D M$ is the collection of all vectors in $T M$ of length less than or equal to 1 . Let int $D M \subset D M$ be the subbundle of tangent vectors of length strictly less than 1 .

Let $\overline{\mathbf{R}}_{D}^{T}(M, g)$ denote the simplicial set whose $k$-simplices are topological disc bundle isomorphisms

$$
\bar{f}: \Delta^{k} \times D M \rightarrow \Delta^{k} \times D M
$$

fixing the zero section, which commute with the projection to $\Delta^{k}$ and cover some homeomorphism $f: \Delta^{k} \times M \rightarrow \Delta^{k} \times M$.

A simplicial subset $\overline{\mathbf{R}}_{D}^{o}(M, g) \subset \overline{\mathbf{R}}_{D}^{T}(M, g)$ is defined by requiring that

$$
\left.\bar{f}\right|_{\Delta^{k} \times \operatorname{int}(D M)}: \Delta^{k} \times D M \rightarrow \Delta^{k} \times D M
$$

is a fiberwise isometry.

The simplicial sets $\underline{\mathbf{R}}_{D}^{o}(M, g)$ and $\underline{\mathbf{R}}_{D}^{T}(M, g)$ are defined similarly but they must cover the identity map on $\Delta^{k} \times M$. Note that the natural inclusion between simplicial sets

$$
\sigma_{D}: \underline{\mathbf{R}}_{D}^{T}(M, g) / \underline{\mathbf{R}}_{D}^{o}(M, g) \rightarrow \overline{\mathbf{R}}_{D}^{T}(M, g) / \overline{\mathbf{R}}_{D}^{o}(M, g)
$$

is a homotopy equivalence, by the same argument given in [BL74, p. 12].

The map $S: \underline{\mathbf{R}}^{T}(M) / \underline{\mathbf{R}}^{o}(M) \rightarrow \mathbf{S} \Gamma\left(\mathcal{B}_{n}(M)\right)$ from Section 2.1 can easily be adapted (see Remark 3 in Appendix) to obtain a corresponding map

$$
S_{D}: \underline{\mathbf{R}}_{D}^{T}(M, g) / \underline{\mathbf{R}}_{D}^{o}(M, g) \rightarrow \mathbf{S} \Gamma\left(\mathcal{B}_{n}^{D}(M)\right) .
$$

Now fix the fiber preserving homeomorphism $\phi: \operatorname{int}(D M) \rightarrow T M$ defined by

$$
\phi(v)=\frac{v}{1-\sqrt{\langle v, v\rangle_{\pi(v)}}} .
$$

We can then form a commutative diagram

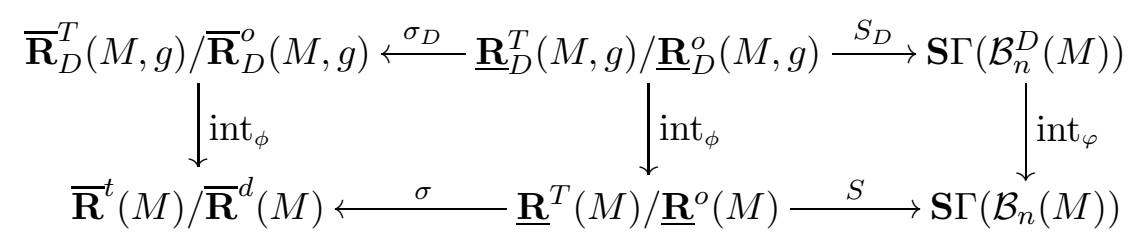

where the vertical maps are induced by restriction of a homeomorphism of $D^{n}$ (or $D M$ ) to its interior and then conjugation by $\varphi$ (or $\phi$ ) and $\sigma$ is the homotopy equivalence (6) of the Appendix below. Taking homotopy groups yields the following commutative diagram 


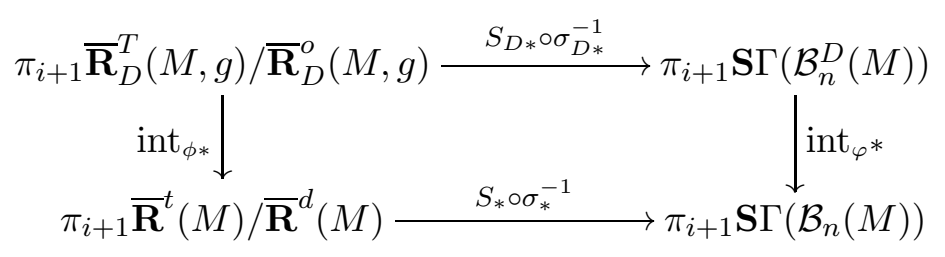

Proof of Lemma 5. Let $f: \Delta^{i+1} \times M \rightarrow \Delta^{i+1} \times M$ be an $(i+1)$-simplex of $\mathbf{T O P}_{0}(M)$ representing an element $[f]$ in $\pi_{i+1}\left(\mathbf{T O P}_{0}(M), \mathbf{D I F F}_{0}(M)\right) \simeq \pi_{i+1} \frac{\operatorname{Top}_{0}(M)}{\operatorname{Diff}_{0}(M)}$, such that $\Phi_{*}^{g}$ 。 $d_{*}[f]=0$, i.e. $f_{z}:=\left.f\right|_{\{z\} \times M} \in \operatorname{Diff}_{0}(M)$ for all $z \in \partial \Delta^{i+1}$ and there exists a continuous map $\Delta^{i+1} \rightarrow \operatorname{MET}^{\mathcal{N C}}(M), z \mapsto g_{z}$, with the property that $g_{z}=\left(f_{z}\right)_{*} g$ for all $z \in \partial \Delta^{i+1}$. We shall exhibit an element in $\pi_{i+1} \Gamma\left(\mathcal{B}_{n}^{D}(M)\right)$ whose image under int ${ }_{\varphi *}$ coincides with $\mu_{*}[f]$ (refer to the notation in Section 2.1.).

Fix a universal cover $\widetilde{M}$ of $M$ and let $\Gamma$ be its group of deck transformations. The homeomorphism $\Delta^{i+1} \times M \rightarrow \Delta^{i+1} \times M$ sending $(z, x)$ to $\left(z, f_{z}(x)\right)$ lifts to a homeomorphism $\Delta^{i+1} \times \widetilde{M} \rightarrow \Delta^{i+1} \times \widetilde{M}$ which in turn restricts to a homeomorphism $\widetilde{f}_{z}: \widetilde{M} \rightarrow \widetilde{M}$ for each $z \in \Delta^{i+1}$.

The universal cover $\widetilde{M}$ acquires an $(i+1)$-parameter family of complete Riemannian metrics $\widetilde{g}_{z}=p^{*} g_{z}$ without conjugate points. Note that for all $z \in \Delta^{i+1}$, there exists a canonical vector bundle isomorphism (over the identity) $\rho_{g_{z}}: T M \rightarrow T M$ such that $\rho_{g_{z}}$ is a fiberwise isometry between the fixed Euclidean structure on $T M$ (i.e. $g$ ) and the one given by the metric $g_{z}$ (see [MS74, p.24]). This can be thought of as an $(i+1)$-simplex $\mathfrak{R} \in \underline{\mathbf{R}}^{d}(M)$ defined by $\mathfrak{R}(z, v)=\left(z, \rho_{g_{z}}(v)\right)$, for all $(z, v) \in \Delta^{i+1} \times T M$.

Let $\exp ^{\tilde{g}}: T \widetilde{M} \rightarrow \widetilde{M} \times \widetilde{M}$ be the exponential map with respect to the metric $\tilde{g}$, i.e. $\exp ^{\tilde{g}}(v)=$ (foot $(v), \gamma_{v}(1)$ ), where $\gamma_{v}$ is the unique geodesic with initial velocity $v$. The corresponding map $\bmod \Gamma$ is denoted by $\exp _{\Gamma}^{g}: T M \rightarrow \widetilde{M} \times_{\Gamma} \widetilde{M}$.

Now for each $z \in \Delta^{i+1}$ define the following map:

$$
\Upsilon_{z}: T M \stackrel{\exp _{\Gamma}^{g}}{\longrightarrow} \widetilde{M} \times_{\Gamma} \widetilde{M} \stackrel{\widetilde{f}_{z} \times_{\Gamma} \widetilde{f}_{z}}{\longrightarrow} \widetilde{M} \times_{\Gamma} \widetilde{M} \stackrel{\left(\exp _{\Gamma}^{g z} \circ \rho_{g_{z}}\right)^{-1}}{\longrightarrow} T M
$$

Because the exponential map of a Riemannian metric on $\widetilde{M}$ without conjugate points is a diffeomorphism, these maps are $\mathbb{R}^{n}$-bundle isomorphisms which cover $f_{z}$. Moreover, when $z \in$ $\partial \Delta^{i+1}$, the map $\Upsilon_{z}$ is a linear isomorphism. Thus we obtain a class $[\Upsilon]$ in the relative homotopy group $\pi_{i+1}\left(\overline{\mathbf{R}}^{t}(M), \overline{\mathbf{R}}^{d}(M)\right)$. Furthermore, by the definition of $\delta$ (see Section 2.1), we have the equation

$$
\delta_{*}[f]=[\mathfrak{R} \circ \Upsilon]=[\mathfrak{R}]+[\Upsilon]=[\Upsilon] \in \pi_{i+1}\left(\overline{\mathbf{R}}^{t}(M), \overline{\mathbf{R}}^{d}(M)\right) \simeq \pi_{i+1} \overline{\mathbf{R}}^{t}(M) / \overline{\mathbf{R}}^{d}(M),
$$

since $[\mathfrak{R}]=0$.

Now we exploit the geometry at infinity of the universal cover of $M$ to construct topological representations of the disc bundle of $M$.

Observe that since $M$ is compact, the metrics $\widetilde{g_{z}}$ are all quasi-isometric to $\widetilde{g}=p^{*} g$, and since the fundamental group of $M$ is hyperbolic we can extend each self-homeomorphism $\widetilde{f}_{z}$ to a self-homeomorphism $\overline{f_{z}}: \bar{M} \rightarrow \bar{M}$ of the geometric compactification $\bar{M}$ of $\widetilde{M}$, whose points at infinity are represented by $g$-quasi-geodesic rays in $\widetilde{M}$ (see [BH99, p. 400-405]).

This yields a homeomorphism

$$
\bar{\Upsilon}_{z}: D M \stackrel{\operatorname{Exp}_{\Gamma}^{g}}{\longrightarrow} \widetilde{M} \times_{\Gamma} \bar{M} \stackrel{\widetilde{f}_{z} \times_{\Gamma} \overline{f_{z}}}{\longrightarrow} \widetilde{M} \times_{\Gamma} \bar{M} \stackrel{\left(\operatorname{Exp}_{\Gamma}^{g_{z}} \circ \rho_{g_{z}}\right)^{-1}}{\longrightarrow} D M
$$


which clearly covers $f_{z}$, and is a fiberwise isometry for $z \in \partial \Delta^{i+1}$. Here $\operatorname{Exp}_{\Gamma}^{g}$ is given by the following map mod $\Gamma$

$$
v \mapsto \begin{cases}\left(\pi(v), \exp _{\pi(v)}^{g}\left(v / 1-\|v\|_{g}\right)\right) & \text { if }\|v\|_{g}<1 \\ \left(\pi(v), \gamma_{v}(\infty)\right) & \text { if }\|v\|_{g}=1,\end{cases}
$$

where $\gamma_{v}(\infty)$ is the point at infinity determined by the geodesic ray $\widetilde{M}$ with initial velocity $v$.

Hence the collection of maps $\bar{\Upsilon}_{z}, z \in \Delta^{i+1}$, determines a class $[\bar{\Upsilon}]$ in $\pi_{i+1}\left(\overline{\mathbf{R}}_{D}^{T}(M, g), \overline{\mathbf{R}}_{D}^{o}(M, g)\right) \simeq$ $\pi_{i+1}\left(\overline{\mathbf{R}}_{D}^{T}(M, g) / \overline{\mathbf{R}}_{D}^{o}(M, g)\right)$ with the property that

$$
\operatorname{int}_{\phi *}[\bar{\Upsilon}]=[\Upsilon]
$$

The result now follows from equation (5) and the commutativity of diagram (4).

\section{Appendix}

Here we prove Lemma 2 and Lemma 3, following ideas from [BL74].

Proof of Lemma Q Consider the following composition

$$
\sigma: \underline{\mathbf{R}}^{T}(M) / \underline{\mathbf{R}}^{o}(M) \rightarrow \underline{\mathbf{R}}^{t}(M) / \underline{\mathbf{R}}^{o}(M) \rightarrow \underline{\mathbf{R}}^{t}(M) / \underline{\mathbf{R}}^{d}(M) \rightarrow \overline{\mathbf{R}}^{t}(M) / \overline{\mathbf{R}}^{d}(M)
$$

induced by the obvious maps. We will prove that $\sigma$ is a homotopy equivalence. The rightmost map is a weak homotopy equivalence by [BL74, Proposition 1.4]). The leftmost map is a weak equivalence by Kister-Mazur theorem. In fact, consider the fiber bundle $\mathcal{E}(M) \rightarrow M$ whose fiber over $x \in M$ is the space $\mathcal{E} \mathcal{M B}\left(\mathcal{U}_{0}, T_{x} M\right)$ of germs of embeddings of $\mathcal{U}_{0}$ into $T_{x} M$ fixing the origin, for some neighbourhood $\mathcal{U}_{0}$ of the origin in $T_{x} M$. Similarly we define the bundle $\mathcal{T}(M) \rightarrow M$ with fiber the space $\operatorname{Top}\left(T_{x} M, 0\right)$ of self-homeomorphisms of $T_{x} M$ fixing the origin. There are obvious simplicial isomorphisms $\underline{\mathbf{R}}^{T}(M) \rightarrow \mathbf{S} \Gamma(\mathcal{T}(M))$ and $\underline{\mathbf{R}}^{t}(M) \rightarrow \mathbf{S} \Gamma(\mathcal{E}(M))$ which make the following diagram commutative

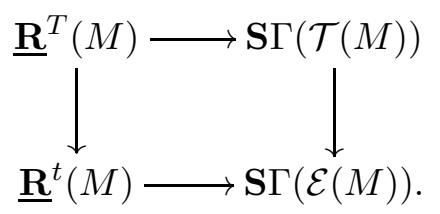

It follows from Kister's theorem that the right vertical map is a weak homotopy equivalence. To see this, note that the restriction of $\mathcal{T}(M) \rightarrow \mathcal{E}(M)$ to an individual fiber is the map $\operatorname{Top}\left(\mathbb{R}^{n}, 0\right) \rightarrow \mathcal{E} \mathcal{M} \mathcal{B}\left(\mathcal{U}_{0}, \mathbb{R}^{n}\right)$. This map factors as the composite $\operatorname{Top}\left(\mathbb{R}^{n}, 0\right) \rightarrow \operatorname{Emb}\left(\mathbb{R}^{n}, 0\right) \rightarrow$ $\mathcal{E} \mathcal{M B}\left(\mathcal{U}_{0}, \mathbb{R}^{n}\right)$ where the middle term is the space of continuous self-embeddings of $\mathbb{R}^{n}$ fixing the origin. Kister Kis64 showed that the first map is a homotopy equivalence. The second map is also a homotopy equivalence as can be seen by first passing to the corresponding simplicial fibration between their singular complexes, and then changing PL-equivalences by continuous embeddings in [KL66, Lemma 1.5. Part c) and e)] to show that the fiber is contractible.

An obstruction theory argument now shows that the map between section spaces is also a weak homotopy equivalence (see for example [BL77, Lemma 3.10]) . Therefore the map on the left is a weak homotopy equivalence.

It is now easy to see that the vertical left map induces the desired weak homotopy equivalence

$$
\underline{\mathbf{R}}^{T}(M) / \underline{\mathbf{R}}^{o}(M) \rightarrow \underline{\mathbf{R}}^{t}(M) / \underline{\mathbf{R}}^{o}(M)
$$


Finally, note that since $\mathrm{O}(n)$ is homotopy equivalent to $G L_{n}(\mathbb{R})$, it also follows that $\underline{\mathbf{R}}^{o}(M) \subset$ $\underline{\mathbf{R}}^{d}(M)$ is a weak homotopy equivalence and hence so is the middle map in (6). This completes the proof.

We now prove Lemma 3 ,

Let $G$ be a topological group and $H$ a closed subgroup of $G$ such that $G \rightarrow G / H$ has local cross sections. For example $G=\operatorname{Top}\left(\mathbb{R}^{n}, 0\right)$ or $\operatorname{Top}\left(D^{n}, 0\right)$ and $H=\mathrm{O}(n)$.

Let $\mathcal{B}_{0}: X \rightarrow B$ be a fiber bundle with fiber $Y$ and structure group $H$. Suppose that action of $H$ on $Y$ extends to an action of $G$ on $Y$, so that we can also we regard $\mathcal{B}_{0}$ as a bundle with structure group $G$.

Let $\underline{\mathbf{R}}^{G}$ be the simplicial set with $k$-simplices given by commutative diagrams

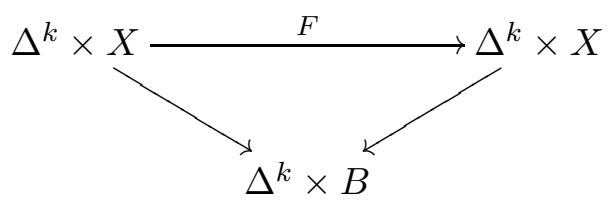

where $F$ is a $G$-equivalecnce, i.e. an isomorphism between bundles with structure group $G$.

Likewise one defines the simplicial set $\underline{\mathbf{R}}^{H}$, and observe that there is an inclusion $\underline{\mathbf{R}}^{H} \subset \underline{\mathbf{R}}^{G}$. Following Burghelea-Lashof [BL74, p.29] and Steenrod [Ste51, p. 44-45], we define a simplicial map

$$
\widehat{S}: \underline{\mathbf{R}}^{G} / \underline{\mathbf{R}}^{H} \rightarrow \mathbf{S} \Gamma\left(P_{H} X \times_{H} G / H\right),
$$

where $P_{H} X \rightarrow B$ is the principal $H$-bundle associated to $\mathcal{B}_{0}$. The map is defined as follows: let $\left\{V_{j}, \phi_{j}\right\}$ be a local coordinate system for $\mathcal{B}_{0}$ and let $\left\{V_{j}, \phi_{j}^{\prime}\right\}$ be the corresponding local coordinate system for $P_{H} X \times_{H} G / H \rightarrow B$.

Given a $k$-simplex $F: \Delta^{k} \times X \rightarrow \Delta^{k} \times X$ of $\underline{\mathbf{R}}^{G}$, for each $t \in \Delta^{k}$ we obtain a map

$$
\phi_{j}^{-1} \circ F_{t} \circ \phi_{j}: V_{j} \times Y \rightarrow V_{j} \times Y
$$

which preserves the first component. Hence it is determined by some map

$$
\lambda_{j}^{t}: V_{j} \rightarrow G,
$$

such that $\phi_{j}^{-1} \circ F_{t} \circ \phi_{j}(x, y)=\left(x, \lambda_{j}^{t}(x) y\right)$.

Let now $\rho: G \rightarrow G / H$ be the quotient map. Then the map

$$
s: \Delta^{k} \times B \rightarrow \Delta^{k} \times P_{H} X \times_{H} \times G / H
$$

defined by $s(t, x)=\phi_{j}^{\prime}\left(x, \rho\left(\lambda_{j}^{t}(x)\right)\right), x \in V_{j}$ is a section of the bundle $\Delta^{k} \times\left(P_{H} X \times_{H} \times G / H\right) \rightarrow$ $\Delta^{k} \times B$, and hence a $k$-simplex of $\mathbf{S} \Gamma\left(P_{H} X \times_{H} G / H\right)$. This gives a map $\underline{\mathbf{R}}^{G} \rightarrow \mathbf{S} \Gamma\left(P_{H} X \times_{H} G / H\right)$ which is independent of choices of $\left\{V_{j}, \phi_{j}\right\}$ and which induces the desired map

$$
\widehat{S}: \underline{\mathbf{R}}^{G} / \underline{\mathbf{R}}^{H} \rightarrow \mathbf{S} \Gamma\left(P_{H} X \times_{H} G / H\right) .
$$

Proposition 8. The map $\widehat{S}$ sends the 0-simplicies of $\underline{\mathbf{R}}^{G} / \underline{\mathbf{R}}^{H}$ into a union, say $\Gamma_{0}\left(P_{H} X \times_{H} G / H\right)$, of path components of $\Gamma\left(P_{H} X \times_{H} G / H\right)$. Furthermore $\widehat{\widehat{S}}: \underline{\mathbf{R}}^{G} / \underline{\mathbf{R}}^{H} \rightarrow \mathbf{S} \Gamma_{0}\left(P_{H} X \times_{H} G / H\right)$ is a simplicial isomorphism.

Assuming this proposition, the proof of Lemma 3 is straightforward:

Proof of Lemma 3. Set $G=\operatorname{Top}\left(\mathbb{R}^{n}, 0\right)$ and $H=\mathrm{O}(n)$ in the previous proposition. Then compose $\widehat{S}$ with the map $\Gamma\left(P_{H} \times{ }_{H} G / H\right) \rightarrow \Gamma\left(\mathcal{B}_{n}(M)\right)$, induced by the inclusion $G \rightarrow \operatorname{Top}\left(\mathbb{R}^{n}\right)=$ $\operatorname{Top}(n)$. 
Remark 3. Now set $G=\operatorname{Top}\left(D^{n}, 0\right)$ and $H=\mathrm{O}(n)$ in the previous proposition and compose $\widehat{S}$ with the map $\Gamma\left(P_{H} \times_{H} G / H\right) \rightarrow \Gamma\left(\mathcal{B}_{n}^{D}(M)\right)$, induced by the inclusion $G \rightarrow \operatorname{Top}\left(D^{n}\right)$. This gives the map $S_{D}$ from Section 5 ,

In order to prove Proposition 8 we restate the theorem in Ste51, p. 45]. First regard $\mathcal{B}_{0}$ as a $Y$-bundle $\mathcal{B}: X \stackrel{\pi}{\rightarrow} B$ with structure group $G$. An $(H, Y)$-bundle structure on $\mathcal{B}$ is an equivalence class of pairs $\left[\left(\mathcal{B}_{1}, F_{1}\right)\right]$

- $\mathcal{B}_{1}$ is a bundle $X_{1} \stackrel{\pi_{1}}{\longrightarrow} B$ with fiber $Y$ and structure group $H$.

- $F_{1}: X_{1} \rightarrow X$ is a $G$-equivalence over $B$.

- Two pairs $\left(\mathcal{B}_{1}, F_{1}\right)$ and $\left(\mathcal{B}_{2}, F_{2}\right)$ are equivalent if and only if there exists an $H$-equivalence $\mathcal{F}: X_{1} \rightarrow X_{2}$ over $B$ such that $F_{2} \circ \mathcal{F}=F_{1}$.

Let $\mathcal{S}_{(H, Y)}(\mathcal{B})$ denote the set of equivalence classes of $(H, Y)$-bundle structures on $\mathcal{B}$. Denote by $\pi^{\prime}: P_{H} X \times_{H} G / H \rightarrow B$ the bundle projection. For each equivalence class $\left[\left(\mathcal{B}_{1}, F_{1}\right)\right]$, note that $F_{1}: X_{1} \rightarrow X$ determines a collection of maps $\left\{\lambda_{j}: V_{j} \rightarrow G\right\}$. Together, they determine a map $B \rightarrow P_{H} X \times_{H} G / H$

$$
x \mapsto \phi_{j}^{\prime}\left(x, \rho\left(\lambda_{j}(x)\right)\right),
$$

for $x \in V_{j}$, which is a section of the bundle $P_{H} X \times_{H} G / H$. Thus we have a map

$$
\mathcal{S}: \mathcal{S}_{(H, Y)}(\mathcal{B}) \rightarrow \Gamma\left(P_{H} X \times_{H} G / H\right) .
$$

Let $q: E H \rightarrow B H$ be the universal $H$-bundle and consider the $H$-bundle map $\bar{h}_{0}: P_{H} X \rightarrow$ $E H$ covering a map $h_{0}: B \rightarrow B H$ which classifies the bundle $P_{H} X \rightarrow B$. Identify $E H$ and $E G$ so that $G$ acts on $E H$ on the right with orbit space $B G$ and let $\hat{h}_{0}: P_{H} X \times_{H} G / H \rightarrow B H$ be given by $[x, \rho(\alpha)] \mapsto q\left(\bar{h}_{0}(x) \cdot \alpha\right)$, for $(x, \alpha) \in P_{H} X \times G$.

Remark 4. $\hat{h}_{0}: P_{H} X \times_{H} G / H \rightarrow B H$ is a $G / H$-bundle map over $i \circ h_{0}: B \rightarrow B G$ where $i: B H \rightarrow B G$ is the quotient map.

Lemma 9. The following holds:

1. $\mathcal{S}$ is a bijection.

2. $B \stackrel{\mathcal{S}\left(\left[\mathcal{B}_{1}, F_{1}\right]\right)}{\longrightarrow} P_{H} \times_{H} G / H \stackrel{\hat{h}_{0}}{\longrightarrow} B H$ is a classifying map for $\mathcal{B}_{1}$.

We now proceed to prove Prosposition 8 assuming Lemma 9 ,

Proof of Prosposition 8. For any 0-simplex $F: X \rightarrow X$ of $\underline{\mathbf{R}}^{G}$ one has $\widehat{S}(F)=\mathcal{S}\left[\left(\mathcal{B}_{0}, F\right)\right]$. So by the first part of Lemma 9, the map $\widehat{S}$ is monic on the 0-simplices. Thus to show the first part of Proposition 8 it suffices to show that if $s_{t}, t \in[0,1]$ is a path in $\Gamma\left(P_{H} X \times_{H} G / H\right)$ such that $s_{0} \in \operatorname{im}(\widehat{S})$, then $s_{1} \in \operatorname{im}(\widehat{S})$. In fact, suppose that $\widehat{S}(F)=s_{0}$ by Lemma 9 there exists $\left[\left(\mathcal{B}_{1}, F_{1}\right)\right]$ such that $\mathcal{S}\left(\left[\mathcal{B}_{1}, F_{1}\right]\right)=s_{1}$. By Part 2. of Lemma 9, since $s_{1}$ is path connected to $s_{0}$, there exists an $H$-equivalence $h: \mathcal{B}_{1} \rightarrow \mathcal{B}_{0}$ (because their classifying maps are homotopic). Hence $\widehat{S}\left(F_{1} \circ h^{-1}\right)=\mathcal{S}\left(\left[\mathcal{B}_{0}, F_{1} \circ h^{-1}\right]\right)=\mathcal{S}\left[\mathcal{B}_{1}, F_{1}\right]=s_{1}$.

This shows the first part of the proposition. The second part follows from a similar argument.

We now prove the Lemma 9 , 
Proof of Lemma 9. The first part is the same as [Ste51, 9.4, p. 44-45]. To show the second part, it suffices to find an $H$-bundle map $P_{H} X_{1} \rightarrow E H$ covering $B \stackrel{\mathcal{S}\left[\mathcal{B}_{1}, F_{1}\right]}{\longrightarrow} P_{H} X \times_{H} G / H \stackrel{\hat{h}_{0}}{\longrightarrow}$ $B H$, where $P_{H} X_{1}$ is the associated principal $H$-bundle of $\mathcal{B}_{1}: X_{1} \rightarrow B$.

Since there is a natural $H$-bundle map $P_{G} X=P_{H} X \times_{H} G \rightarrow E H$ given by $[x, \alpha] \mapsto \bar{h}_{0}(x) \cdot \alpha$, covering $\hat{h}_{0}: P_{H} X \times_{H} G / H \rightarrow B H$; we only need to give an $H$-bundle map $P_{H} X_{1} \rightarrow P_{G} X$ covering the map $B \stackrel{\mathcal{S}\left[\mathcal{B}_{1}, F_{1}\right]}{\longrightarrow} P_{H} X \times_{H} G / H$.

This map is defined via the following commutative diagram

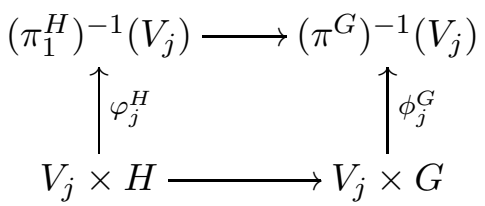

where $\left\{V_{j}, \phi_{j}^{G}\right\}$ is a local coordinate system for the principal $G$-bundle $\pi^{G}: P_{G} X \rightarrow B$, and $\left\{V_{j}, \phi_{j}^{H}\right\}$ is a local coordinate system for the principal $H$-bundle $P_{H} X_{1} \stackrel{\pi_{1}^{H}}{\longrightarrow} B$. The lower horizontal map is given by $(x, h) \mapsto\left(x, \lambda_{j}(x) h\right)$ and $\left\{\lambda_{j}: V_{j} \rightarrow G\right\}$ is induced by $F_{1}$.

\section{References}

[BFJ18] Mauricio Bustamante, Francis Thomas Farrell, and Yi Jiang. On negatively curved bundles with hyperbolic fibers outside the igusa stable range. Mathematische Annalen, May 2018.

[BH99] Martin R. Bridson and André Haefliger. Metric spaces of non-positive curvature, volume 319 of Grundlehren der Mathematischen Wissenschaften [Fundamental Principles of Mathematical Sciences]. Springer-Verlag, Berlin, 1999.

[BK72] A. K. Bousfield and D. M. Kan. Homotopy limits, completions and localizations. Lecture Notes in Mathematics, Vol. 304. Springer-Verlag, Berlin-New York, 1972.

[BL74] Dan Burghelea and Richard Lashof. The homotopy type of the space of diffeomorphisms. I, II. Trans. Amer. Math. Soc., 196:1-36; ibid. 196 (1974), 37-50, 1974.

[BL77] D. Burghelea and R. Lashof. Stability of concordances and the suspension homomorphism. Ann. of Math. (2), 105(3):449-472, 1977.

[BL12] Arthur Bartels and Wolfgang Lück. The Borel conjecture for hyperbolic and CAT(0)-groups. Ann. of Math. (2), 175(2):631-689, 2012.

[BLR08] Arthur Bartels, Wolfgang Lück, and Holger Reich. The $K$-theoretic Farrell-Jones conjecture for hyperbolic groups. Invent. Math., 172(1):29-70, 2008.

[Far02] F. T. Farrell. The Borel conjecture. In Topology of high-dimensional manifolds, No. 1, 2 (Trieste, 2001), volume 9 of ICTP Lect. Notes, pages 225-298. Abdus Salam Int. Cent. Theoret. Phys., Trieste, 2002.

[FH78] F. T. Farrell and W. C. Hsiang. On the rational homotopy groups of the diffeomorphism groups of discs, spheres and aspherical manifolds. In Algebraic and geometric topology (Proc. Sympos. Pure Math., Stanford Univ., Stanford, Calif., 1976), Part 1, Proc. Sympos. Pure Math., XXXII, pages 325-337. Amer. Math. Soc., Providence, R.I., 1978.

[FJ89] F. T. Farrell and L. E. Jones. Compact negatively curved manifolds (of dim [unk] 3,4) are topologically rigid. Proceedings of the National Academy of Sciences, 86(10):3461-3463, 1989.

[FJ93] F. T. Farrell and L. E. Jones. Topological rigidity for compact non-positively curved manifolds. In Differential geometry: Riemannian geometry (Los Angeles, CA, 1990), volume 54 of Proc. Sympos. Pure Math., pages 229-274. Amer. Math. Soc., Providence, RI, 1993.

[FO09] F. Thomas Farrell and Pedro Ontaneda. The Teichmüller space of pinched negatively curved metrics on a hyperbolic manifold is not contractible. Ann. of Math. (2), 170(1):45-65, 2009.

[FO10a] F. Thomas Farrell and Pedro Ontaneda. On the topology of the space of negatively curved metrics. $J$. Differential Geom., 86(2):273-301, 2010. 
[FO10b] Tom Farrell and Pedro Ontaneda. Teichmüller spaces and negatively curved fiber bundles. Geom. Funct. Anal., 20(6):1397-1430, 2010.

[FO15] F. T. Farrell and P. Ontaneda. The space of nonpositively curved metrics of a negatively curved manifold. J. Differential Geom., 99(2):285-311, 2015.

[Gro87] M. Gromov. Hyperbolic groups. In Essays in group theory, volume 8 of Math. Sci. Res. Inst. Publ., pages 75-263. Springer, New York, 1987.

[Igu88] Kiyoshi Igusa. The stability theorem for smooth pseudoisotopies. K-Theory, 2(1-2):vi+355, 1988.

[Igu02] Kiyoshi Igusa. Higher Franz-Reidemeister torsion, volume 31 of AMS/IP Studies in Advanced Mathematics. American Mathematical Society, Providence, RI; International Press, Somerville, MA, 2002.

[Kis64] J. M. Kister. Microbundles are fibre bundles. Ann. of Math. (2), 80:190-199, 1964.

[KL66] N. H. Kuiper and R. K. Lashof. Microbundles and bundles. II. Semisimplical theory. Invent. Math., 1:243-259, 1966.

[Kli74] Wilhelm Klingenberg. Riemannian manifolds with geodesic flow of Anosov type. Ann. of Math. (2), 99:1-13, 1974.

[KM63] Michel A. Kervaire and John W. Milnor. Groups of homotopy spheres. I. Ann. of Math. (2), 77:504-537, 1963.

[KS77] Robion C. Kirby and Laurence C. Siebenmann. Foundational essays on topological manifolds, smoothings, and triangulations. Princeton University Press, Princeton, N.J.; University of Tokyo Press, Tokyo, 1977. With notes by John Milnor and Michael Atiyah, Annals of Mathematics Studies, No. 88.

[Lle85] Irene Llerena. Localization of fibrations with nilpotent fibre. Math. Z., 188(3):397-410, 1985.

[Mil64] J. Milnor. Microbundles. I. Topology, 3(suppl. 1):53-80, 1964.

[Ml87] Jesper Michael Mø ller. Nilpotent spaces of sections. Trans. Amer. Math. Soc., 303(2):733-741, 1987.

[MS74] John W. Milnor and James D. Stasheff. Characteristic classes. Princeton University Press, Princeton, N. J.; University of Tokyo Press, Tokyo, 1974. Annals of Mathematics Studies, No. 76.

[Rug94] Rafael O. Ruggiero. Expansive dynamics and hyperbolic geometry. Bol. Soc. Brasil. Mat. (N.S.), 25(2):139-172, 1994

[Rug03] Rafael Oswaldo Ruggiero. On the divergence of geodesic rays in manifolds without conjugate points, dynamics of the geodesic flow and global geometry. Astérisque, (287):xx, 231-249, 2003. Geometric methods in dynamics. II.

[Ste51] Norman Steenrod. The Topology of Fibre Bundles. Princeton Mathematical Series, vol. 14. Princeton University Press, Princeton, N. J., 1951.

[Wal78] Friedhelm Waldhausen. Algebraic K-theory of topological spaces. I. In Algebraic and geometric topology (Proc. Sympos. Pure Math., Stanford Univ., Stanford, Calif., 1976), Part 1, Proc. Sympos. Pure Math., XXXII, pages 35-60. Amer. Math. Soc., Providence, R.I., 1978.

\author{
Mauricio Bustamante \\ Institut FÜr Mathematik, Universität Augsburg \\ EMAIL: Mauricio.BustamanteLondono@math.uni-augsburg.de \\ Francis Thomas FARRELL \\ Yau Mathematical Sciences Center, Tsinghua University, Beijing, China \\ EMAIL: farrell@math.tsinghua.edu.cn \\ YI JIANG \\ Yau Mathematical Sciences Center, Tsinghua University, Beijing, China \\ EMAIL: yjiang117@mail.tsinghua.edu.cn
}

